Case Report

\title{
Systemic Light Chain Amyloidosis (Congo Red Inconclusive) with Underlyng Clonal Expansion Not Meeting the Criteria of Light Chain Monoclonal Gammopathy of Renal Significance or Light Chain Myeloma: A Case Report
}

\author{
Farsana Mustafa', $\underline{\text { Sanjay Kumar }}$, Vijay Kumar \\ ${ }^{1}$ Post-Graduate Student, ${ }^{2}$ Associate Professor, Department of Medicine, Lady Hardinge Medical College, New Delhi, India. \\ ${ }^{3}$ Professor, Department of Pathology, Atal Bihari Vajpayee Institute of Medical Sciences, New Delhi, India. \\ DOI: https://doi.org/10.24321/2349.7181.202019
}

I $\quad \mathbf{N} \quad \mathbf{F} \quad \mathbf{O}$

Corresponding Author:

Sanjay Kumar, Department of Medicine, Lady Hardinge Medical College, New Delhi, India.

E-mail Id:

drsanjaykumar_30@yahoo.com

Orcid Id:

https://orcid.org/0000-0001-5688-4376

How to cite this article:

Mustafa F, Kumar S, Kumar V. Systemic Light Chain Amyloidosis (Congo Red Inconclusive) with Underlyng Clonal Expansion Not Meeting the Criteria of Light Chain Monoclonal Gammopathy of Renal Significance or Light Chain Myeloma: A Case Report. J Adv Res Med 2020; 7(4): 15-19.

Date of Submission: 2021-02-18

Date of Acceptance: 2021-03-05

\section{$\begin{array}{llllllll}\mathbf{A} & \mathbf{B} & \mathbf{S} & \mathbf{T} & \mathbf{R} & \mathbf{A} & \mathbf{C} & \mathbf{T}\end{array}$}

Fifty-eight-year-old male admitted for evaluation of nephrotic syndrome and chronic diarrhoea was detected to have Immunoglobulin light chain amyloidosis (AL Amyloidosis) which was congo red inconclusive from renal biopsy. Bone marrow biopsy showed monoclonal plasma cells of $40 \%$ and light chain assay showed predominance of immunoglobulin lambda light chain. The diagnosis was neither fitting into the current diagnostic criteria for light chain Monoclonal Gammopathy of Renal Significance (MGRS) nor light chain myeloma. Literature is scarce regarding patients with $\mathrm{AL}$ amyloidosis having underlying clonal expansion not meeting the criteria of light chain myeloma or light chain MGRS.

Keywords: Amyloidosis, Congo Red Negative, MGRS, Light Chain Myeloma

\section{Introduction}

Amyloidosis are a group of diseases characterized by extracellular deposition of misfolded proteins in various organs. AL amyloidosis is the most common form with an estimated incidence between 3 and 12 cases per million persons per year. ${ }^{1}$ Even though $\mathrm{AL}$ amyloidosis is not defined as a classical myeloma defining event, outcome of patients with $\mathrm{AL}$ amyloidosis (AL-PCMM) who have clonal bone marrow plasmacytosis $\geq 10$ percent are comparable to patients meeting criteria for coexisting Multiple myeloma. ${ }^{2}$ Light chain myeloma constitutes approximately $15 \%$ of patients with multiple myeloma and it has a poorer prognosis compared to IgA or IgG variant of myeloma. A 
rare but very serious comorbidity of LCMM is systemic light chain amyloidosis, which is observed in 5 to $10 \%$ of cases of LCMM. ${ }^{3}$ MGRS (Monoclonal Gammopathy of Renal Significance) was a terminology introduced by International Kidney and Monoclonal Gammopathy Research Group (IKMG) to describe haematological conditions that produce a monoclonal immunoglobulin associated kidney injury. According to literatures low clonal burden, who are not meeting the criteria for MM or other malignancies are included under MGRS. But unlike MGUS, these clones can cause vital organ damage in the form of neuropathy, cardiomyopathy, hepatic dysfunction and dermopathy. ${ }^{4}$ There are some disease states which may lie in between the spectrum of MGRS and frank multiple myeloma which are still in grey zone. We report a case of $\mathrm{AL}$ amyloidosis which lies in this zone of exceptions and clonal expansion associated with $\mathrm{AL}$ amyloidosis which was producing only lambda light chain in excess.

\section{Case Report}

On august $15^{\text {th }} 2020$, a 58 year old male was admitted for evaluation of anasarca and chronic diarrhoea of one and a half years duration. Initially, he noticed swelling of both lower limbs associated with facial puffiness but without any decrease in urine output. He used to have watery loose stools 4 to 6 episode per day and got relieved by over-the-counter medications. He also had bleeding per rectum 5 episodes over last 2 months. There was no history of mucous or pus in stools. No history of fever, weight loss or loss of appetite. He was a chronic smoker with a pack year of 30 years with a background history of chronic obstructive airway disease (severity: category B). There were no similar complaints in any of the other family members. On examination patient had bilateral pitting pedal edema along with facial puffiness. The pulse rate 86 beats per minute and was of a regular rhythm. Blood pressure was 110/ $70 \mathrm{mmHg}$ with no postural drop, a respiratory rate of 22 breaths per minute, which was predominantly abdominothoracic. Abdomen examination revealed a distended abdomen with an everted umbilicus. Shifting dullness was present. Chest examination revealed a stony dull note on percussion in bilateral infraaxillary and infrascapular areas suggesting pleural effusion. A fine inspiratory crepitations was heard in bilateral axillary areas with absent breath sounds in bilateral infraaxillary and infrascapular areas.

\section{Investigation}

The haemoglobin was $12.6 \mathrm{~g} / \mathrm{dL}$, total leucocyte counts $6000 / \mathrm{mm} 3$ with differential count of P68/L30/M1/E1, platelet count 2.4 lakh/mm3, MCV 95 fl, MCH 31.7 pg/cell and $\mathrm{MCHC} 33.3 \mathrm{~g} / \mathrm{dL}$. The peripheral smear was normal without any rouleaux formation. ESR was $22 \mathrm{~mm} / \mathrm{hr}$, blood urea $27 \mathrm{mg} / \mathrm{dL}$, serum creatinine $0.88 \mathrm{mg} / \mathrm{dL}$, uric acid
$4.32 \mathrm{mg} / \mathrm{dL}$, calcium $7.5 \mathrm{mg} / \mathrm{dL}$ (Corrected Calcium 9.36 $\mathrm{mg} / \mathrm{dL}$ ) Phosphorous $3.36 \mathrm{mg} / \mathrm{dL}$, total Protein $4.82 \mathrm{~g} / \mathrm{dL}$, albumin $1.74 \mathrm{~g} / \mathrm{dL}$, globulin $3.1 \mathrm{~g} / \mathrm{dL}$, ALP $91 \mathrm{IU} / \mathrm{L}$, estimated glomerular filtration rate was $124 \mathrm{ml} / \mathrm{min} / 1.73$, sodium $135 \mathrm{mmol} / \mathrm{L}$ and potassium $3.6 \mathrm{mmol} / \mathrm{L}$. As there was no micronutrient deficiencies or peripheral lymphopenia protein loss was expected to be from urine rather than a gastrointestinal cause. His Chest x-ray (Figure 1) showed right sided encysted pleural effusion with bilateral lower zone heterogenous opacity and his COVID 19 RT PCR was negative.

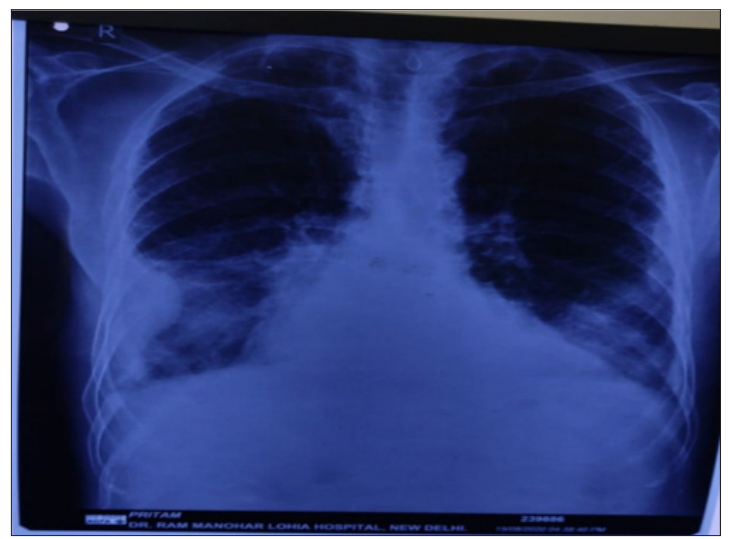

Figure I.Chest X-ray P/A view: showing bilateral lower zone heterogenous opacity with right sided encysted pleural effusion

Ultrasound chest revealed echogenic right pleural fluid with few septations. Ultrasound guided pleural tap showed 250 cells with $80 \%$ mononuclear, $20 \%$ polymorphs, glucose 56 $\mathrm{mg} / \mathrm{dL}$, protein $1.3 \mathrm{gm} / \mathrm{L}$, pleural fluid CBNAAT and ADA were negative, LDH $240 \mathrm{U} / \mathrm{L}$ (serum LDH $170 \mathrm{U} / \mathrm{L}$, pleural: serum $L D H$ ratio $>0.6)$, gram stain revealed gram positive cocci however culture didn't grow any organism. Ascitic tap was transudative. As a part of workup for chronic diarrhoea HIV, HBsAg and anti-HCV ELISA was done and found to be negative. The stool examination for ova and cyst was negative and thyroid profile, vitamin B12 and folate levels were normal. Ultrasound whole abdomen revealed normal liver echotexture and span of $14 \mathrm{~cm}$ with moderate ascites. The bilateral renal size was normal with maintained corticomedullary differentiation. Electrocardiogram revealed low voltage complexes in limb leads. 2D Echocardiogram revealed normal valves and chambers with left ventricular ejection fraction of $60 \%$. There was grade 1 left ventricular diastolic dysfunction, with thickening of intraventricular septum without any sparkly appearance and tissue Doppler parameters indicating a restrictive pathology. There was no regional wall motion abnormality. His serologic cardiac biomarkers were normal except for Brain Natriuretic Peptide (BNP) which was higher than his age-related cutoff. In this context it was inconclusive as any fluid overload condition can cause a rise in it. Cardiac MRI was not done 
due to financial constraints of family and a delayed date from hospital due to covid pandemic. His urine routine microscopy with dipstick was showing 3+ protein, urine albumin-creatinine ratio was 3203 mg/gm which was in nephrotic range, a 24-hr urine protein of $14 \mathrm{gm}$ per day. Urine Bence Jones protein was negative. The $\mathrm{HbA} 1 \mathrm{C}, \mathrm{C} 3$ and C4 levels were normal. ANA, extended ANA, cANCA, pANCA and anti-GBM antibodies were negative. Immunoglobulin levels IgG was lower than reference limit i.e $481 \mathrm{mg} / \mathrm{dl}$ (ref: 540-1822) while IgA 131 mg/dl (ref: 63-484) and IgM $68 \mathrm{mg} / \mathrm{dl}$ (ref: 22-240) were in normal limits. Lipid profile revealed a triglyceride level of $309 \mathrm{mg} / \mathrm{dL}$ (elevated) while total cholesterol, HDL and LDL cholesterol were normal. Proctoscopy and local examination of rectum was normal. We proceeded with CECT chest which showed moderate to gross pleural effusion with loculations and underlying atelectasis of bilateral lungs, diffuse cystic changes with emphysema in bilateral lungs predominantly in upper lobes secondary to COPD. CECT whole abdomen revealed moderate ascites, thickened and oedematous gall bladder walls likely reactionary cholecystitis secondary to ascites and bilateral renal cortical cyst. CT enterography was normal. Colonoscopy was done and it showed multiple large pliable ulcers with intervening normal mucosa involving terminal ileum. The caecum, ascending, transverse and descending colon was normal. A biopsy was taken from the ulcerated site. Amoebic serology was normal and biopsy from ulcer revealed lamina propria with mild chronic inflammatory infiltrate and mild edema. No evidence of cryptitis, crypt abscess or granuloma. Upper GI endoscopy was normal. As a part of workup of nephrotic syndrome with IgG deficiency we did a renal biopsy which showed $A L$ amyloidosis showing lambda restriction involving glomeruli, blood vessels and interstitium (Figures 2 to 5). However, the amyloid was congo red inconclusive.

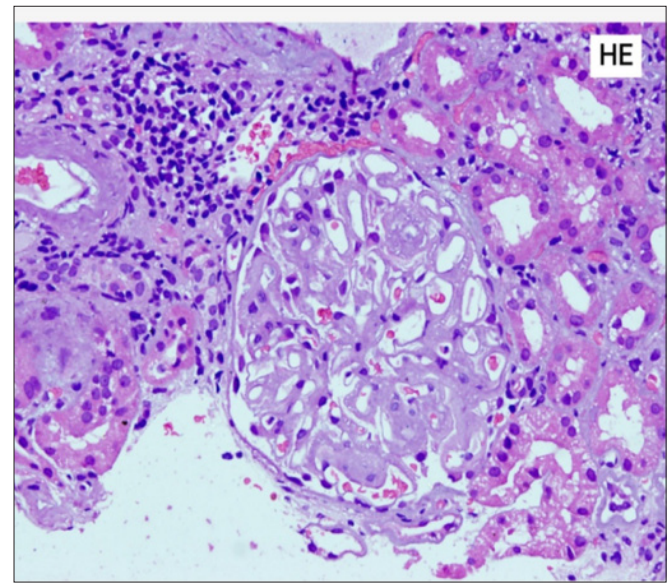

Figure 2.Light microscopy (H\&E stain) of glomeruli high power view showing viable glomeruli enlarged due to deposition of pink amorphous material in mesangium, peripheral capillary walls

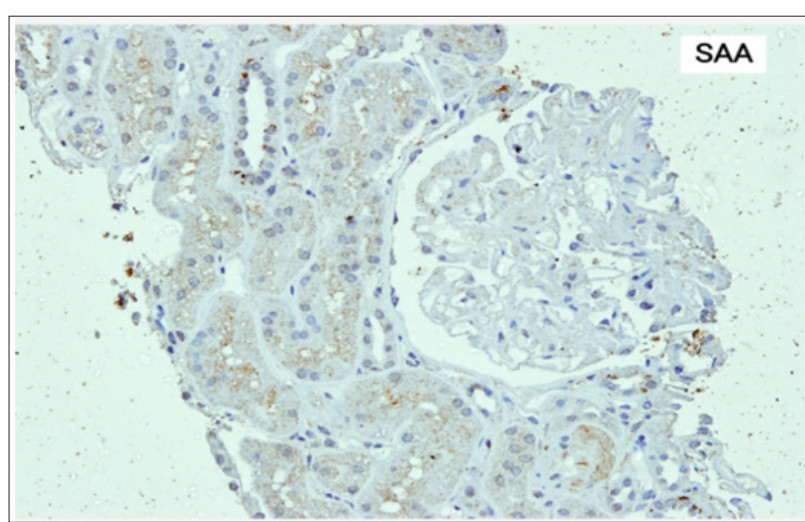

Figure 3.Immunohistochemistry for SAA amyloid is negative

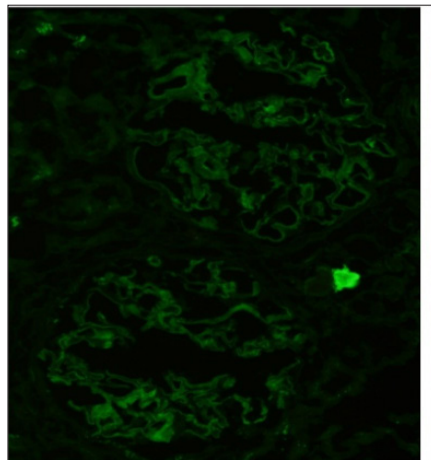

Kappa

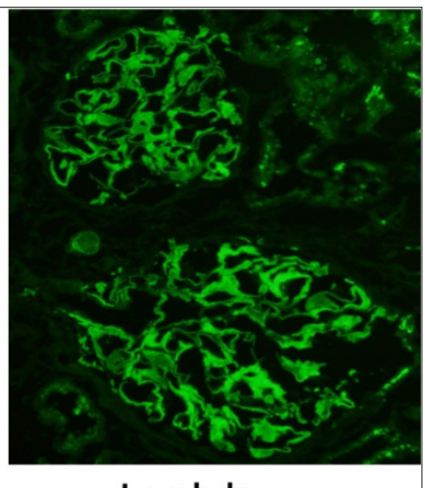

Lambda
Figure 4.Immunofluorescence staining of glomeruli showing fluffy deposits along the peripheral capillary walls of mesangium with predominance for lambda

light chains over kappa light chains (lambda 2+, kappa 0)
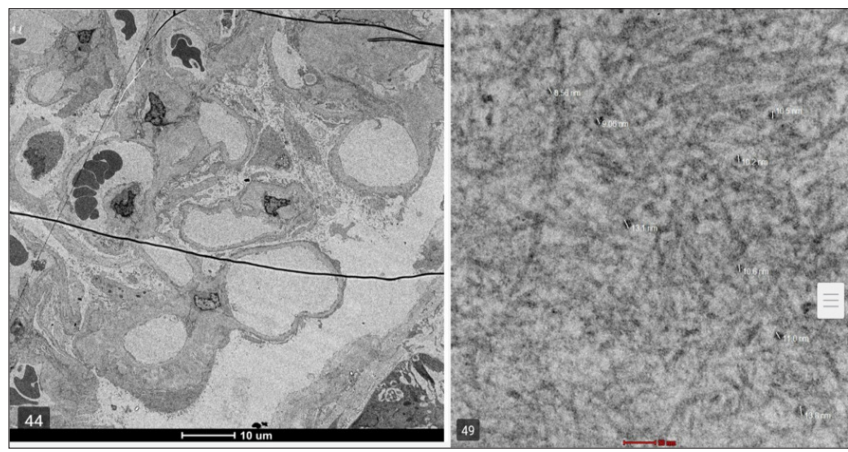

Figure 5.Scanning Electron Micrograph of glomeruli showing haphazardly arranged numerous fine fibrils of mean diameter $9.73 \mathrm{~nm}$ identified in peripheral capillary walls and mesangium

We proceeded with a serum protein electrophoresis (Figure 6) which didn't show any M spike. Light chain assay showed beta 2 microglobulin 5560 ng/ ml (609-2366), free lambda levels of $532 \mathrm{mg} / \mathrm{L}$ (ref: 8.3-27), free kappa $22 \mathrm{mg} / \mathrm{L}$ (ref: 6.722.4), free kappa/lambda ratio 0.041 (ref: 0.31-1.5), serum involved to uninvolved free light chain ratio was 24.18, Immunofixation showed faint $\mathrm{M}$ band in lambda region 
while absent in kappa region. Urine protein electrophoresis was not done as albumin was excreted in urine due to deposition of amyloid material in glomerular filtration barrier. Bone marrow biopsy revealed a hypocellular bone marrow with numerous typical and atypical plasma cells (identified by positive for CD38). These cells comprise $40 \%$ of total cell population. Bone marrow aspirate flow cytometry showed plasma cell marker positivity. With these findings we did a skeletal survey which was not showing any bony lytic lesions (Figure7). Patient was started on intravenous antibiotics and diuretics. A repeat pleural fluid analysis revealed a transudative picture with gram stain culture sensitivity negative.

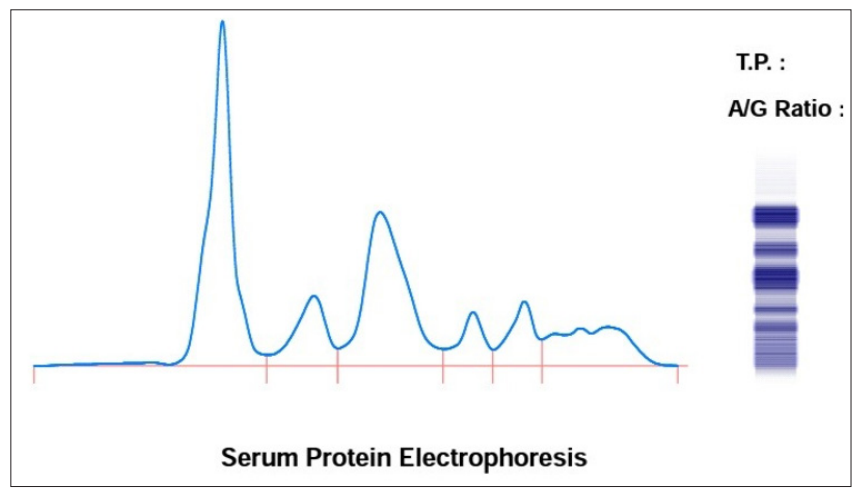

Figure 6.Serum Protein electrophoresis: showing increase in alpha I, alpha 2 globulin and distortion in gamma region along with evidence of hypoproteinemia and hypoalbuminemia. No $M$ band seen
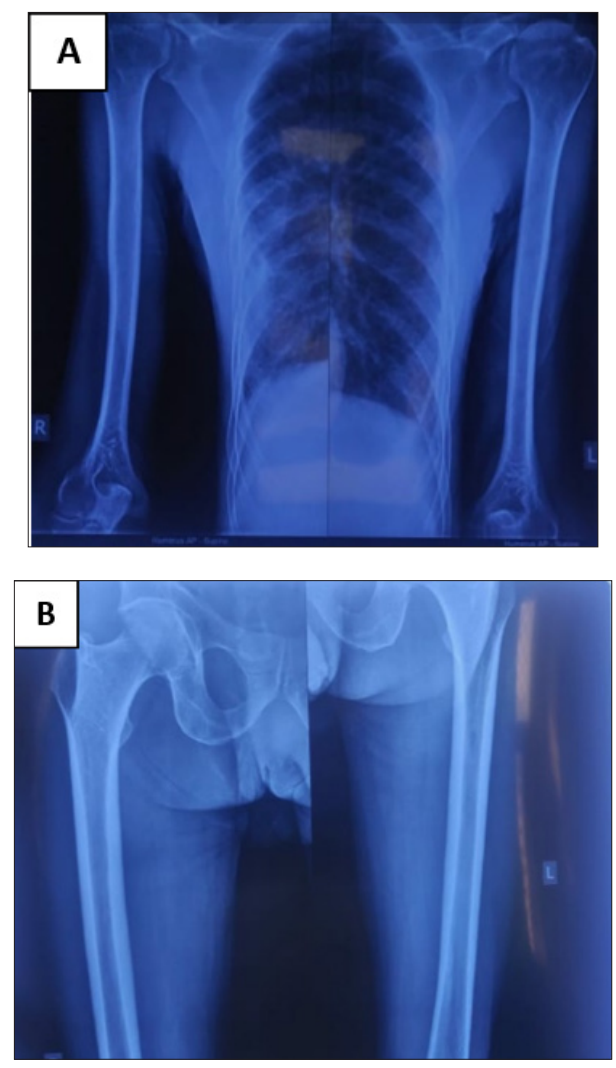

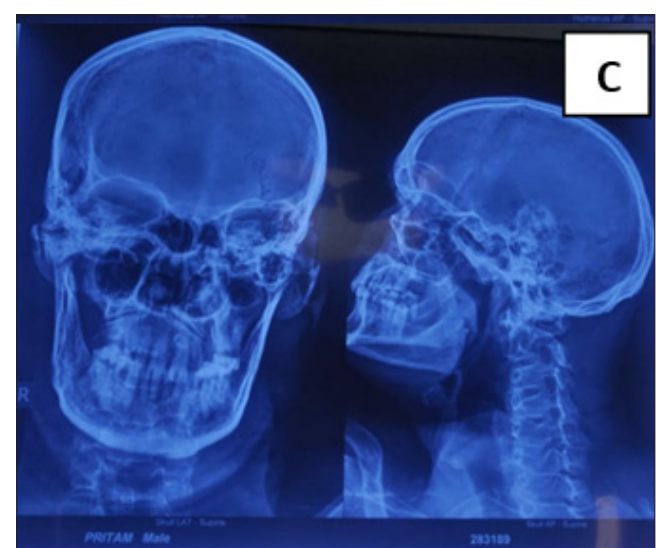

Figure 7.A, B, C: skeletal x-rays: did not reveal any bony lytic lesions

\section{Discussion}

Immunoglobulin light chain amyloidosis or primary amyloidosis are characterized by deposition of fibrils composed of fragments of monoclonal light chains. Affected patients may have amyloidosis alone or underlying spectrum of plasma cell dyscracias (for example Monoclonal Gammopathy of Undetermined Significance, Multiple myeloma or waldenstrom macroglobulinemia). Differentiation of $\mathrm{AL}$ amyloidosis from other forms of localized amyloidosis and other types of monoclonal immunoglobulin deposition disease is vital as clinical course and therapy are markedly different. In this case even though congo red staining was inconclusive (probably due to various errors such as gauge of renal biopsy needle, extended time of fixation in carnoy's solution, lack of proper staining techniques, microscope with proper strain free optics and various other technical errors), ${ }^{5}$ the electron microscopy showed solid nonbranching and randomly arranged fibrils of diameter 7-12 nm size which was conclusive of amyloidosis. Indirect immunofluorescence showed lambda light chain predominance over kappa light chains.

Light Chain Multiple Myeloma (LCMM) is the third most common type of $\mathrm{MM}$ and carries a poor prognosis and renal involvement is seen more commonly in LCMM as compared to other types of multiple myeloma. ${ }^{3}$ Clonal plasma cells synthesize large number of light chains which aggregate from tissues to form insoluble fibrils that form amyloid and get deposited in multiple organs (in our case in kidney, cardiac and probably gastrointestinal involvement). Patients secreting lambda light chains have comparatively shorter survival than those secreting kappa chains, the exact cause of this is not known. ${ }^{6}$ Revised International Myeloma Working Group diagnostic criteria 2014 definition of multiple myeloma defines involved/ uninvolved serum free light chain ratio $>100$ and involved serum free light chain concentration $\geq 10 \mathrm{mg} / \mathrm{dl}$ as a myeloma defining event. Henceforth integrated clonal cells producing light chain excess also into the standard definition for Myeloma. ${ }^{7}$ Cast 
nephropathy involving distal tubules which presents as renal insufficiency is the classical myeloma defining event but $\mathrm{AL}$ amyloidosis presenting as nephrotic syndrome and severe hypoalbuminemia is not included. ${ }^{8}$ MGRS is defined as clonal proliferative disorder that produces nephrotoxic monoclonal immunoglobulin, but does not meet haematological criteria for a specific malignancy. In such diseases clonal burden should be low $<10 \%$ monoclonal bone marrow plasma cells and monoclonal immunoglobulin $<30 \mathrm{~g} / \mathrm{L}$. MGRS clones were also known to produce vital organ damages apart from kidney. In our case monoclonal plasma cells in bone marrow was $40 \%$, involved to uninvolved light chain ratio was 24: 18 and serum monoclonal light chain was $532 \mathrm{mg} / \mathrm{L}$. So, our patient was in a bridge zone of continual spectrum of MGRS and MM from where more clonal expansion can occur and can progress into Multiple Myeloma. As per literature, systemic light chain amyloidosis with multiple myeloma defined either by CRAB or by more than $10 \%$ bone marrow plasma cell clones have poor prognosis and for all practical purposes considered as a single group. ${ }^{2}$ Chemotherapy targeting the clones should be selected weighing it against the risk of drug toxicities and treatment related mortality without any delay.

\section{Conclusion}

Multiple myeloma and $\mathrm{AL}$ amyloidosis are caused by clonal plasma cell proliferation in bone marrow. So, establishing clonal plasma cell percentage in bone marrow is vital step in management. Spectrum of disorders in between light chain MGRS and light chain myeloma should be dealt with utmost caution in clinical practice as literature is scarce regarding them. Early diagnosis, proper prognostication and timely risk adapted therapeutic strategies may also be required in such cases without any delay.

\section{Conflict of Interest: None}

\section{References}

1. Quock T, Yan T, Chang E et al. Epidemiology of $A L$ amyloidosis: a real-world study using US claims data. Blood advances 2018; 2(10): 1046-1053.

2. Kourelis T, Kumar S, Gertz M et al. Coexistent Multiple Myeloma or Increased Bone Marrow Plasma Cells Define Equally High-Risk POPULATINS in Patients With Immunoglobulin Light Chain Amyloidosis. Journal of clinical oncology 2013; 31(34): 4319-4324.

3. Rafae A, Malik M N, Abu Zar M et al. An Overview of Light Chain Multiple Myeloma: Clinical Characteristics and Rarities, Management Strategies and Disease Monitoring. Cureus 2018; 10(8): 3148.

4. Leung N, Bridoux F, Batuman V et al. The evaluation of monoclonal gammopathy of renal significance: a consensus report of the International Kidney and Monoclonal Gammopathy Research Group. Nature
Reviews Nephrology 2019; 15: 45-59.

5. Bowen K, Shah N, Lewin M. AL Amyloidosis Presenting with Negative Congo Red Staining in the Setting of High Clinical Suspicion: A Case Report. Case Reports in Nephrology 2012; 2012 Article ID 593460.

6. Pavan M, Ashwani K, Suratkal L et al. Complete remission of lambda light chain myeloma presenting with acute renal failure following treatment with bortezomib and steroids. Indian J Nephrology 2010; 20(2): 94-96.

7. Rajkumar V. Updated Diagnostic Criteria and Staging System for Multiple Myeloma. American Society of Clinical Oncology Educational Book 2016; 36: e418-e423.

8. Suzuki K. Diagnosis and treatment of multiple myeloma and $A L$ amyloidosis with focus on improvement of renal lesion. Clin Exp Nephrol 2012; 16(5): 659-671. 